\title{
Improving data quality for 3D electron diffraction (3DED) by Gatan Image Filter
}

\author{
Taimin Yang, Hongyi Xu, Xiaodong Zou
}

\author{
Department of Material and Environmental Chemistry, Stockholm University, Stockholm, Sweden \\ taimin.yang@mmk.su.se
}

Electrons interact with matter $10^{6}$ times stronger than X-rays do, which makes it an ideal radiation source for diffraction and imaging experiments on submicron- and nano-sized crystals. During the last three decades, 3D electron diffraction (3D ED) has been developed into a regular and reliable technique for structure determination, which is complementary to single-crystal X-ray diffraction (SCXD) and single particle analysis. One issue for electron diffraction is inelastic scattering, which brings background in the diffraction patterns. This background is most obvious for electron diffraction patterns from protein crystals, especially at low angles. Even though modern diffraction data software (XDS, DIALS, MOSFLM) has sophisticated background removal algorithms to deal with this, the existence of inelastic scattering will still add errors in the diffraction experiment. The inelastically scattered electrons can be removed by energy filters. Here, we implemented energy-filtered 3D ED using a Gatan Energy Filter (GIF) in both TEM selected area electron diffraction mode and STEM micro/nanoprobe mode. We explained the setup in detail and this implementation can allow researcher to have better accessibility to energy-filtered 3DED experiments because more microscopes are equipped with a GIF than an in-column omega filter. We also proposed a crystal tracking method in STEM mode using live HAADF image stream. This method enables us to collect energy-filtered 3DED datasets in STEM mode with a larger tilt range without foregoing any frames. This can avoid crystal moving out of the beam during the tilting and the tilt range can always reach the maximum tilt range of the microscope (in our case $\sim 150^{\circ}$ ). We acquired multiple datasets from different crystals and we further processed and refined the structures. We observed that the final $\mathrm{R}_{1}$ will improve $20 \%$ to $30 \%$ for energy-filtered datasets compared with unfiltered datasets. We also discussed the possible reasons that lead to the improvement.

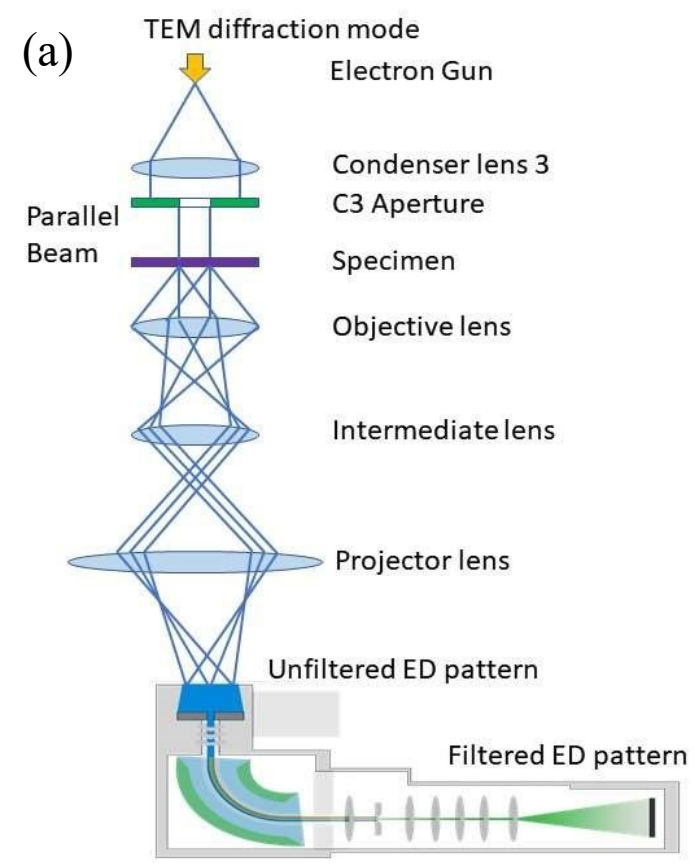

Gatan Image Filter (GIF)

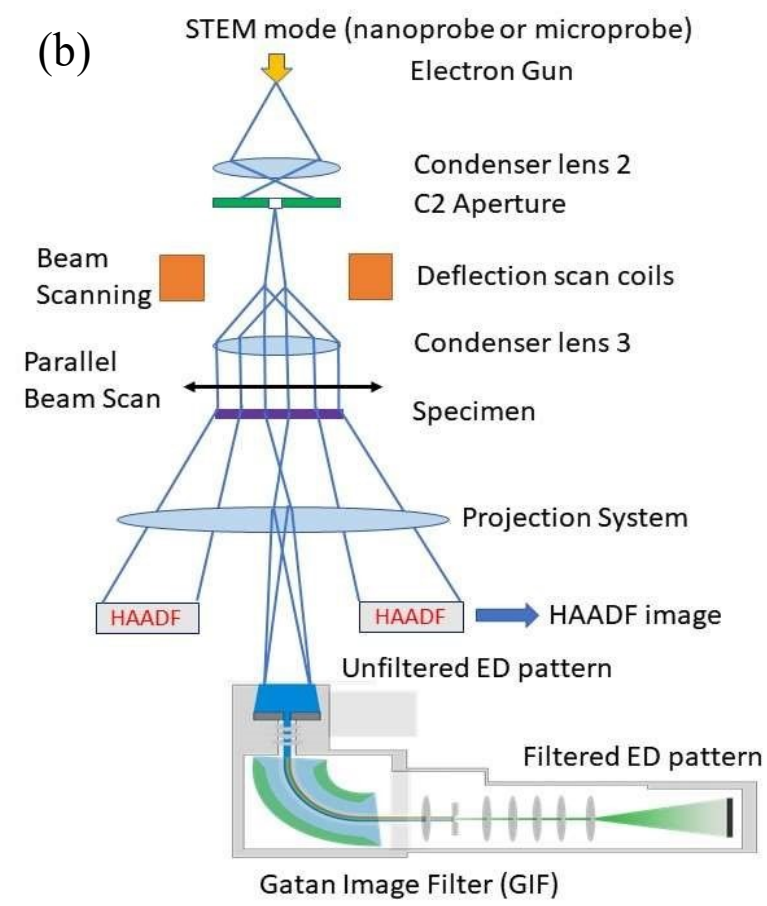

Figure 1. Implementation of Energy filtered 3DED in (a) TEM mode (b) STEM mode. In STEM mode, crystal tracking using live STEM-HAADF image stream can be activated. 


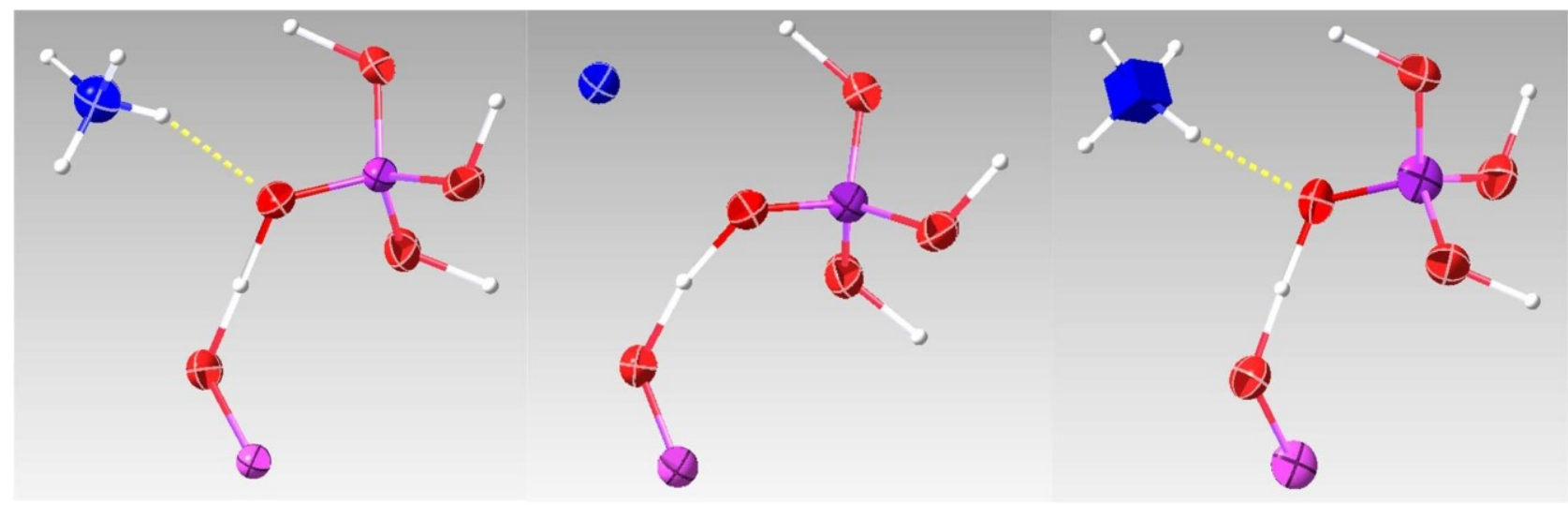

Figure 2. (a) A typical $\mathrm{NH}_{4} \mathrm{H}_{2} \mathrm{PO}_{4}$ crystal structure representation from a filtered dataset. All hydrogen atoms were found and all the ADPs are reasonable after anisotropic refinement (b, c) Two $\mathrm{NH}_{4} \mathrm{H}_{2} \mathrm{PO}_{4}$ crystal structure representations from unfiltered datasets. The refinement was not able to find all the hydrogen atoms around nitrogen atom or unable to obtain reason thermal parameters for the nitrogen atom. The dotted line represents the ionic bond between hydrogen atom and oxygen atom. White for hydrogen; blue for nitrogen; purple for phosphate and red for oxygen.
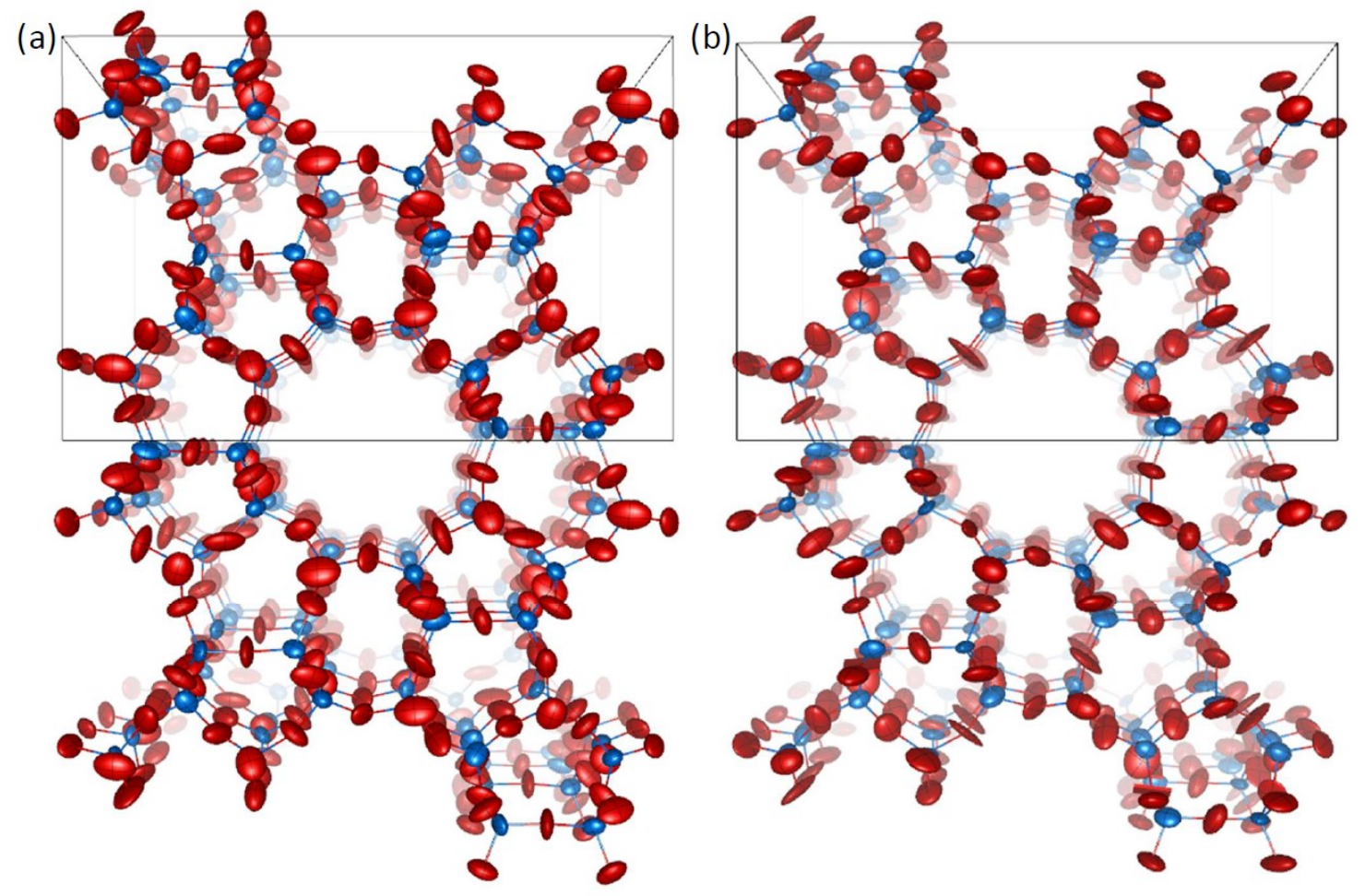

Figure 3. Refined structures of ZSM-5 from (a) filtered dataset (b) unfiltered dataset, showing atomic displacement parameters for the $\mathrm{Si}$ and $\mathrm{O}$ atoms at the $60 \%$ probability level along $\mathrm{b}$ axis. Red for oxygen atoms and blue for silicon atoms. The structure from unfiltered dataset contained a lot of unreasonable ADPs. Some of them became negative ADPs while the ADPs of structure from filtered dataset were reasonable and closer to isotropic state.

Keywords: GIF, energy-filtered 3D electron diffraction, energy-filtered MicroED, STEM HAADF, crystal tracking 